\title{
Terahertz Wave Approach and Application on FRP Composites
}

\author{
Kwang-Hee Im, ${ }^{1}$ David K. Hsu, ${ }^{2}$ Chien-Ping Chiou, ${ }^{2}$ \\ Daniel J. Barnard, ${ }^{2}$ Jong-An Jung, ${ }^{3}$ and In-Young Yang ${ }^{4}$ \\ ${ }^{1}$ Department of Automotive Engineer, Woosuk University, Chonbuk 565-701, Republic of Korea \\ ${ }^{2}$ Center for Nondestructive Evaluation, Iowa State University, Ames, IA 50011, USA \\ ${ }^{3}$ Department of Mechanical and Automotive Engineering, Songwon University, Gwangju 502-210, Republic of Korea \\ ${ }^{4}$ Department of Mechanical Design Engineering, Chosun University, Gwangju 501-759, Republic of Korea
}

Correspondence should be addressed to Kwang-Hee Im; khim@woosuk.ac.kr

Received 27 October 2012; Accepted 12 January 2013

Academic Editor: Abbas Milani

Copyright (c) 2013 Kwang-Hee Im et al. This is an open access article distributed under the Creative Commons Attribution License, which permits unrestricted use, distribution, and reproduction in any medium, provided the original work is properly cited.

\begin{abstract}
Terahertz (THz) applications have emerged as one of the most new powerful nondestructive evaluation (NDE) techniques. A new T-ray time-domain spectroscopy system was utilized for detecting and evaluating orientation influence in carbon fiber-reinforced plastics (CFRPs) composite laminates. Investigation of terahertz time-domain spectroscopy (THz-TDS) was made, and reflection and transmission configurations were studied as a nondestructive evaluation technique. Here, the CFRP composites derived their excellent mechanical strength, stiffness, and electrical conductivity from carbon fibers. Especially, the electrical conductivity of the CFRP composites depends on the direction of unidirectional fibers since carbon fibers are electrically conducting while the epoxy matrix is not. In order to solve various material properties, the index of refraction $(n)$ and the absorption coefficient $(\alpha)$ are derived in reflective and transmission configurations using the terahertz time-domain spectroscopy. Also, for a 48-ply thermoplastic polyphenylene-sulfide-(PPS-) based CFRP solid laminate and nonconducting materials, the terahertz scanning images were made at the angles ranged from $0^{\circ}$ to $180^{\circ}$ with respect to the nominal fiber axis. So, the images were mapped out based on the electrical field (E-field) direction in the CFRP solid laminates. It is found that the conductivity $(\sigma)$ depends on the angles of the nominal axis in the unidirectional fiber.
\end{abstract}

\section{Introduction}

Increasingly, the terahertz radiation has been recognized for their importance in technological applications. Recently, Tray (terahertz ray) advances of technology and instrumentation has provided a probing field on the electromagnetic spectrum because the terahertz radiation has a shorter wavelength and relatively higher resolution than microwaves. The terahertz radiation is of critical importance in the spectroscopy evaluation of airport security screening, medical imaging, polar liquids, industrial systems, and composites as well [1]. Also, the terahertz time-domain spectroscopy (THz-TDS) is leading noncontact accurate detection of flaws and impact damages in composites, in which the THz-TDS is based on photoconductive switches, which rely on the production of few-cycle terahertz pulses using a femtosecond laser to excite a photoconductive antenna [2]. This can generate subpicosecond bursts of $\mathrm{THz}$ radiation and subsequently detect them with high signal-to-noise ratio. With the emitted power distributed over several terahertz, they consequently span a very broad bandwidth. A transient change of the emitter occurs in the resistance of a photoconductive switch on a terahertz timescale $[3,4]$. Also, another method is optical heterodyne conversion, or photomixing, which can be obtained using two continuous-wave (CW) lasers $[5,6]$. The mixing of two lasers could produce beating, which can modulate the conductance of a photoconductive switch by the terahertz difference frequency. So, the $\mathrm{CW}$-terahertz $(\mathrm{CW}-\mathrm{THz})$ radiation is produced. So, the chemical property of materials is of great interest, and the study presents many challenges. At the $\mathrm{THz}$ fundamental level, the problem is one of describing the overall properties of CFRP composite materials. In some cases, T-ray images can show chemical compositions of the object. These features of T-ray imaging have generated interest in commercial applications in diverse 


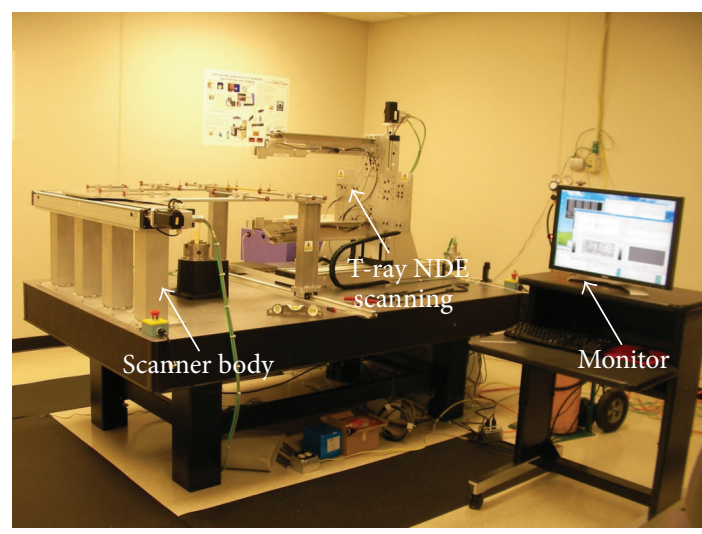

FIGURE 1: THz TDS system for imaging and material parameter measurement.

areas as moisture analysis, quality control of plastic parts, and packaging inspection (monitoring) [7]. In this paper, we report our initial investigations of the index of refraction $(n)$ and the absorption coefficient $(\alpha)$, the electrical conductivity of epoxy matrix composites containing carbon fibers, and the THz scan images of CFRP solid laminates. Since carbon fibers are electrically conducting while the epoxy matrix is not, the CFRP composites are strongly anisotropic. The $\mathrm{THz}$ fundamental equations were derived in order to solve the index of refraction $(n)$ and the absorption coefficient $(\alpha)$ in the general nonconducting materials. The dependence of the effective conductivity on the fiber orientation is examined. The data confirm that these composites can be described by a conductivity model with principal axes along and normal to the nominal fiber axes. Thus, the $\mathrm{THz}$ effective scan images can be made according to the angels at the orientation of carbon fibers. It follows that the higher contrast images are being governed by the $E$-field direction. Also, it was found that the best scanning angles were optimized based on the $E$ field direction to the function of fiber angles.

\section{Theory and Experiment}

The THz TDS setup is shown in Figure 1 in order to collect the material parameter data and perform the images. A modelocked, $100 \mathrm{fs}$, Ti: sapphire laser drove a photoconductive switch using a lock-in amplifier. The $\mathrm{THz}$ pulse was reflected at $17^{\circ}$ from the sample and was detected using an electro-optic technique (Za Te crystal).

2.1. Terahertz System. In the past years, T-ray technology has received more interest and attention because of its unique properties as a nondestructive evaluation (NDE) tool. The $\mathrm{THz}$ frequency region represents an important intersection between spatial resolution and penetration depth. The terahertz systems used in this research were provided by TeraView Limited. The instrumentation includes a timedomain spectroscopy (TDS) pulsed system and a frequencydomain continuous-wave (CW) system. The TDS techniques for generating, manipulating, and detecting terahertz pulses have been immerged. By obtaining images with THz-TDS, in

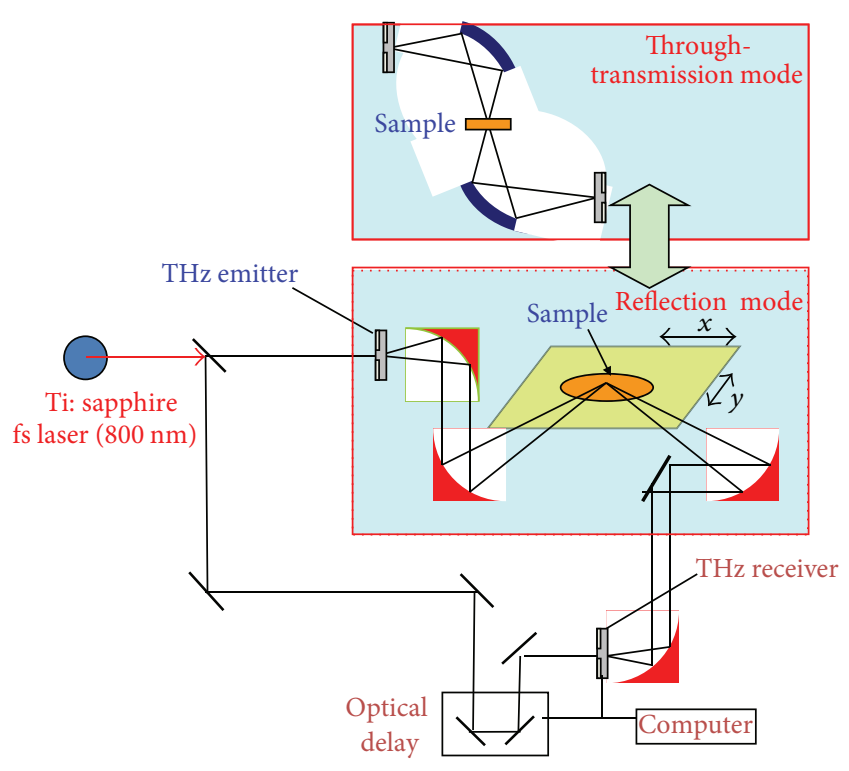

FIGURE 2: A schematic diagram of a THz-TDS spectrometer.

which the entire terahertz waveform is measured at each pixel of the image, the data acquisition rate could be increased. The TDS system has a frequency range of $50 \mathrm{GHz}-4 \mathrm{THz}$ and a fast delay line up to $300 \mathrm{ps}$. The beam is focused to focal lengths of $50 \mathrm{~mm}$ and $150 \mathrm{~mm}$, and the full width at half maximum (FWHM) beam widths are, respectively, $0.8 \mathrm{~mm}$ and $2.5 \mathrm{~mm}$. The TDS system can be configured for through-transmission or reflection (small angle pitch-catch) measurements. The frequency range of the CW system is $50 \mathrm{GHz}-1.5 \mathrm{THz}$, with the best resolution being $100 \mathrm{MHz}$. The focal lengths of the CW system are also $50 \mathrm{~mm}$ and $150 \mathrm{~mm}$. Both the TDS and the CW systems are fully fiber optics connected. Figure 2 shows a schematic diagram of a THzTDS spectrometer, used for T-ray reflection and throughtransmission modes.

2.2. Reflection Mode. This method was to determine the index of refraction used to calculate the optical path length difference between the front and back reflections in the time domain. A diagram showing the geometry of the two $\mathrm{THz}$ signals is shown in Figure 3. The refractive index $(n)$ is now defined by

$$
n^{4}-A n^{2}-A \sin ^{2} \theta_{p 1}=0
$$

Here, $A=\left(T^{2} V_{\text {air }}^{2}\right) /\left(4 d_{2}^{2}\right)$, where $T$ is the transmission time of the sample, $d_{2}$ is the sample thickness, $V_{\text {air }}$ is the light speed in air, and $\theta_{p 1}$ is the incident angle of the sample.

2.3. Through-Transmission Mode. Time of flight could be solved without sample as follows:

$$
t_{\text {total time without sample }}=\frac{L}{V_{\text {air }}},
$$


TABLE 1: Average THz refractive indices of the material studied.

\begin{tabular}{lccc}
\hline Materials & Refractive index $(n)^{*}$ & \multicolumn{2}{c}{ Refractive index $(n)$} \\
& & Through-transmission mode & $1.62 \pm 0.03$ \\
PMMA & $1.60 \pm 0.08$ & $1.61 \pm 0.07$ & $1.94 \pm 0.05$ \\
Fused quartz & $1.95 \pm 0.05$ & $1.95 \pm 0.05$ & $2.12 \pm 0.05$ \\
\hline GFRP & - & $2.13 \pm 0.05$ & Reflection mode \\
\hline
\end{tabular}

${ }^{*}$ Known data by $[8,9]$.

where $t_{\text {total time without sample }}$ is the time from the transmitter to the receiver, $L$ is the distance between the transmitter and the receiver, and $V_{\text {air }}$ is the air speed $\left(3 \times 10^{10} \mathrm{~cm} / \mathrm{s}\right)$. Consider

$$
t_{\text {total time with sample }}=\frac{L-d}{V_{\text {air }}}+\frac{d}{V_{s}},
$$

where $t_{\text {total time with sample }}$ is the time from the transmitter to the receiver, $V_{s}$ is the T-ray speed of sample, and $d$ is the thickness of sample.

Penetration time $(\Delta t)$ in order to calculate the index of refraction $(n)$ could be calculated as follows:

$$
\Delta t=t_{\text {total time with sample }}-t_{\text {total time without sample }} \text {. }
$$

In through-transmission mode, the index of refraction (n) could be obtained from the following as shown in Figure 4:

$$
n=1+\frac{\Delta t V_{\mathrm{air}}}{d}
$$

where $\Delta t$ is the difference time between with sample and without sample, $d$ is the sample thickness, $V_{\text {air }}$ is the light speed in air, and $L$ is the distance between pulsed emitter and pulsed receiver.

A method to determine the absorption coefficient $(\alpha)$ was also developed as a conventional way. In throughtransmission mode, the pulses were captured from two samples with different thickness, and the absorption coefficient $(\alpha)$ could be developed by a sample proportional equation from Figures 5(a) and 5(b). The absorption coefficient $(\alpha)$ could then be calculated using the following formula as shown in Figure 5:

$$
\alpha=-\frac{\ln \left(I_{2} / I_{1}\right)}{d_{1}-d_{2}},
$$

where $d_{2}$ is the thicker sample, $d_{1}$ is the thinner sample, $I_{2}$ is the transmitted E-field in the thicker sample, and $I_{1}$ is the transmitted $E$-field in the thinner sample. A goal of this research was to determine if the refractive index of various materials could be calculated with a THz-TDS system in reflection and through-transmission modes. In addition to such samples, a CFRP composite laminate was prepared to assess the utility of a THz TDS system for NDE of orientation in carbon fibers. Another sample consisting of one ply laminated piece was prepared to try to detect a flaw at the back side in order to evaluate the effect of $E$-field and to investigate the backside flaw with $\mathrm{THz}$ TDS imaging.

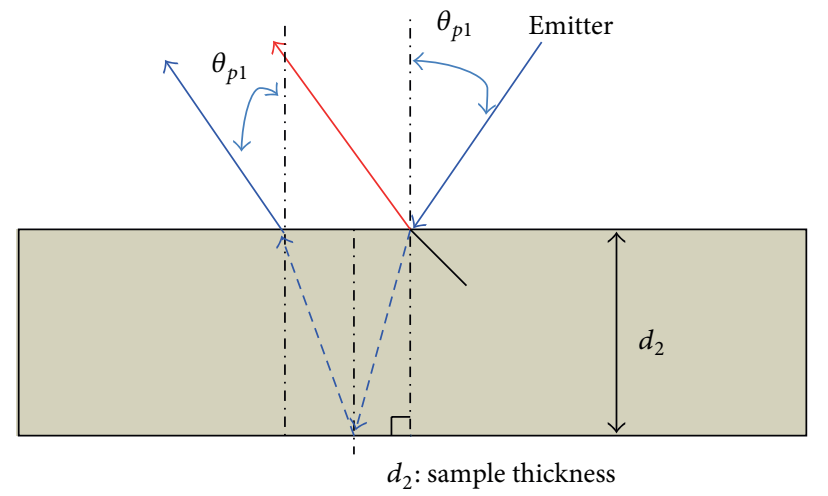

FIGURE 3: Diagram showing the geometry of the surface reflection mode.

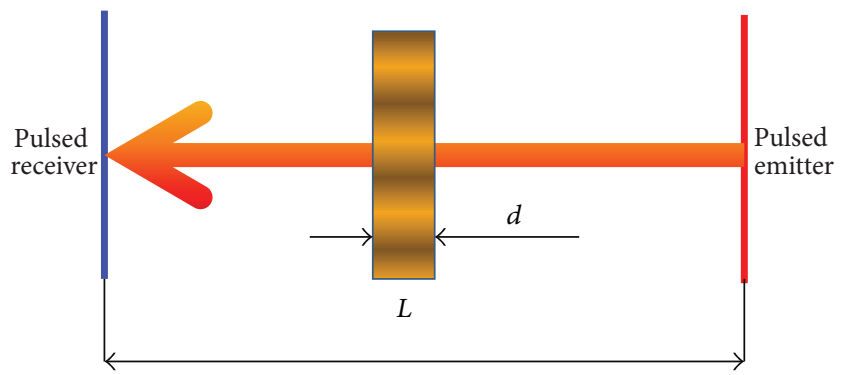

FIgURE 4: Diagram showing the geometry of the through-transmission mode.

\section{Results and Discussion}

3.1. Measurement of Refractive Index. In preparation for measuring material parameters, we first measured a $\mathrm{THz}$ pulse reflected from 24-ply woven glass epoxy GFRP composites. In Figure 6, one can clearly see the initial reflection from the surface and the subsequent reflection off the back surface. A method to determine the index of refraction was used to calculate the optical path length difference using the reflection and through-transmission mode as shown in Table 1. It is found that those data were agreed with the known data for two modes.

3.2. Nonconducting Composites. Terahertz waves can readily penetrate nonconducting materials and can therefore be applied to the nondestructive testing (NDT) of glass, quartz, or Kevlar fiber-reinforced polymer matrix composites. Solid laminates and sandwich structures with honeycomb or foam 


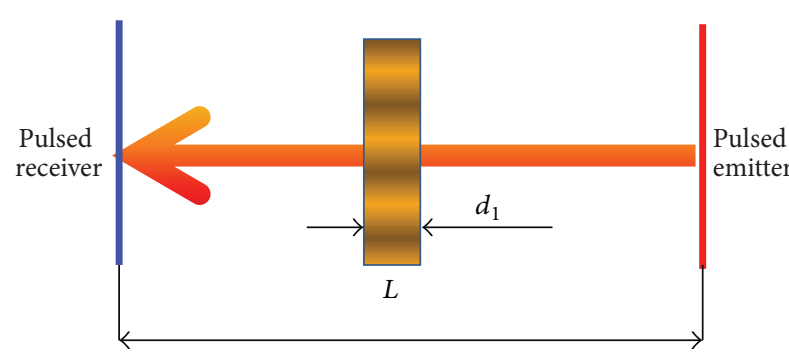

(a)

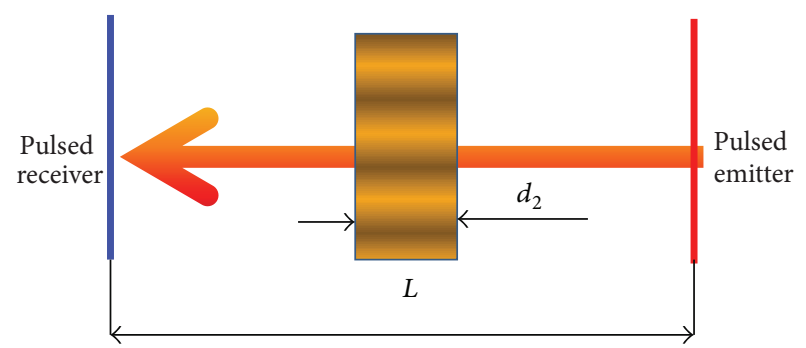

(b)

FIGURE 5: Diagram showing the geometry of the through-transmission mode with two samples in (a) thinner and (b) thicker samples.

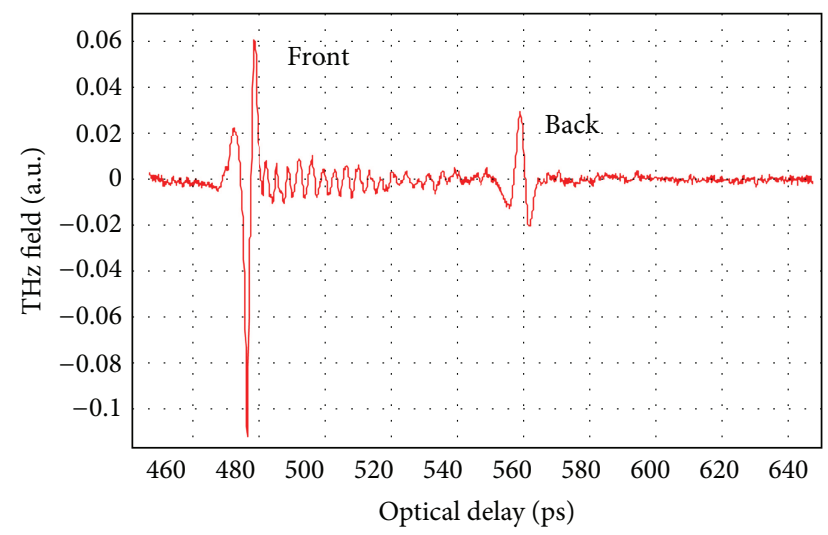

Figure 6: THz TDS pulses reflected from the front and back surfaces.

core can all be examined with terahertz waves. In this study, terahertz radiation was explored for the NDT of a variety of non-conducting composite materials and structures. Some preliminary results are summarized later.

3.2.1. Imaging of Multiple Delaminations. The time-domain waveforms of terahertz pulses in the TDS mode bear strong resemblance to ultrasonic signals. Wave propagation concepts such as time of flight (TOF), transmission and reflection coefficients, refraction, and diffraction are common to both waves. However, there are also fundamental differences when materials are probed with terahertz radiation, an electromagnetic wave, and with ultrasound, a mechanical wave. First and foremost, terahertz waves, unlike ultrasound, do not require a material medium to support it and can therefore readily go through vacuum or air. In the ultrasonic inspection of flaws in a solid, the probing field suffers from the "shadow effect," where a smaller flaw behind a larger flaw could not be detected. In contrast, there is no such limitation with terahertz waves. To demonstrate this effect, two semicircular saw slots were cut parallel to the surfaces into the side of a $28.7 \mathrm{~mm}$ thick woven glass composite laminate to serve as simulated delaminations, as shown in Figure 7. The smaller slot (101.6 mm diameter) was located below the larger slot (127.0 $\mathrm{mm}$ diameter). Using the TDS terahertz system in the through-transmission mode, a scan image was made of the sample containing the double saw slots. The image of the transmitted terahertz pulse amplitude, also shown in Figure 6, clearly revealing both saw slots results demonstrated that terahertz waves do not suffer from "shadow effect." Figures 7(a), 7(b), and 7(c) show the dimensions of sample, Cscan image, and B-scan image, respectively, and TOF means a time of flight in the T-ray. Especially, it is well agreed that a shorter TOF is corresponding with thinner thickness because of two saw cuts.

The sample was also scanned from the top surface with reflection mode TDS terahertz pulse, and the double saw slots were imaged.

3.2.2. Imaging of Impact Damage in Solid Laminates. Based on the capability described easlier, the multiple delaminations in solid composite laminates were imaged with reflection mode TDS terahertz waves. The specimen was a 24 -ply woven glass epoxy solid laminate, and the impact was made with a $50.8 \mathrm{~mm}$ diameter tup at an impact energy of 16 Joules. The reflection mode TDS scan was made on the back side of the sample so that the smaller delaminations were shadowed by the larger and shallower delaminations. Figure 8 shows the time-domain signal (terahertz A-scan trace), where the two prominent pulses were reflections from the top and bottom surfaces of the specimen. To produce the images of the impact-induced delaminations, a time gate was imposed on the A-scan signal, and the peak amplitude within the time gate was used to generate the images. Figure 8 shows four of such images with the time gates placed at $43-69$ ps, 47$69 \mathrm{ps}$, and 56-69 ps, respectively. The series of images clearly showed the decreasing size of the delaminations toward the impact side.

3.2.3. Imaging of Embedded Flaws in Kevlar/Nomex Sandwich. Both the reflection mode (small angle pitch-catch) and the transmission mode TDS terahertz scans were found effective in mapping out defects embedded between the Kevlar composite facesheet and the Nomex honeycomb core of a sandwich panel (skin: $0.38 \mathrm{~mm}$ Kevlar, $12.7 \mathrm{~mm}$ Nomex honeycomb, and cell size: $5.08 \mathrm{~mm}$ ). In addition to using the peak amplitude of the terahertz pulse reflected from or transmitted through the panel, images can also be generated 


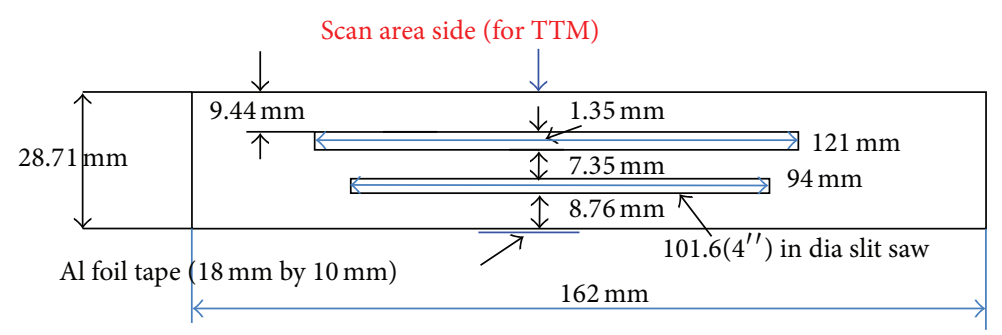

(a) GFRP sample

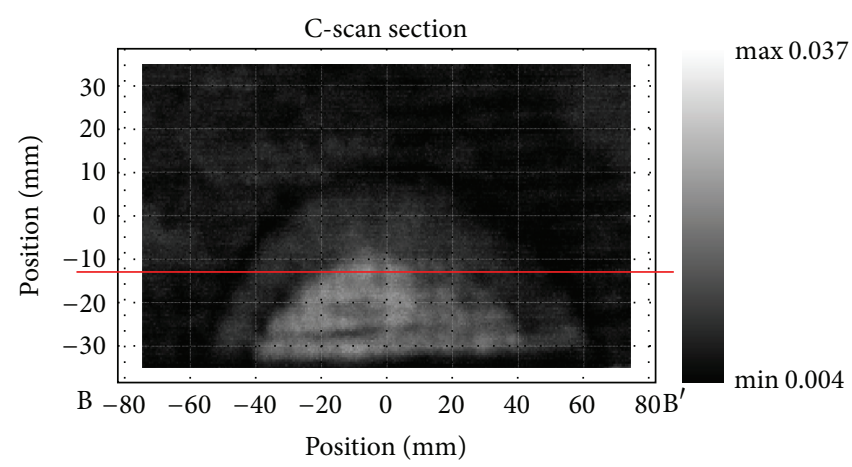

(b) C-scan image

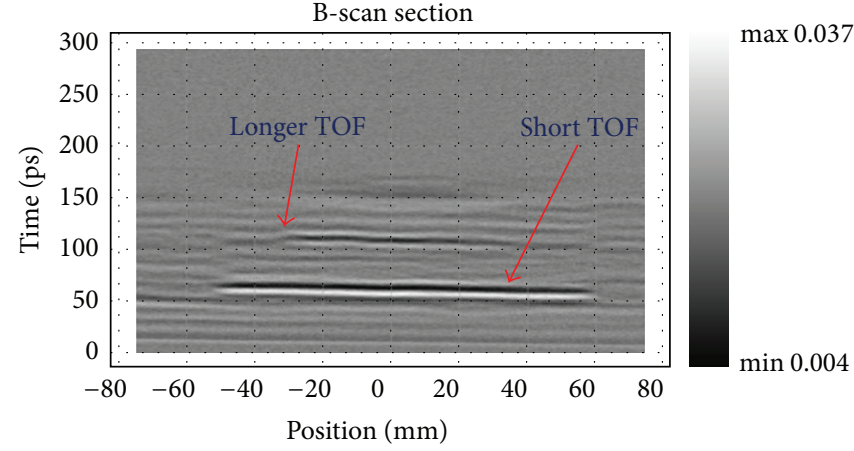

(c) B scan at B-B'

FIGURE 7: Terahertz TDS through-transmission scan image of double saw slots in a glass composite solid laminate. The results demonstrated that terahertz waves do not suffer from "shadow effect."

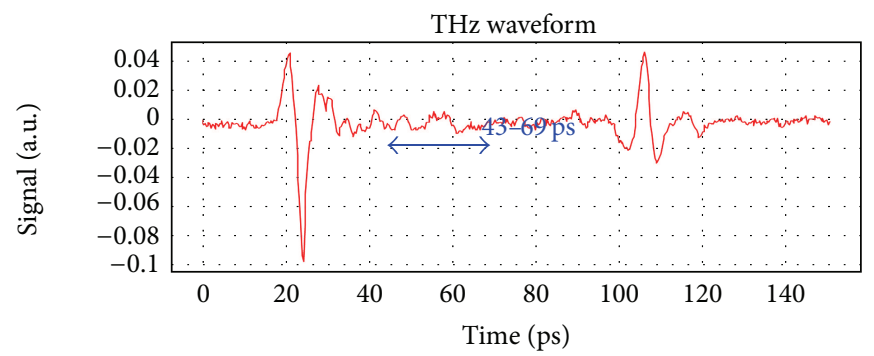

(a) T-ray A-scan

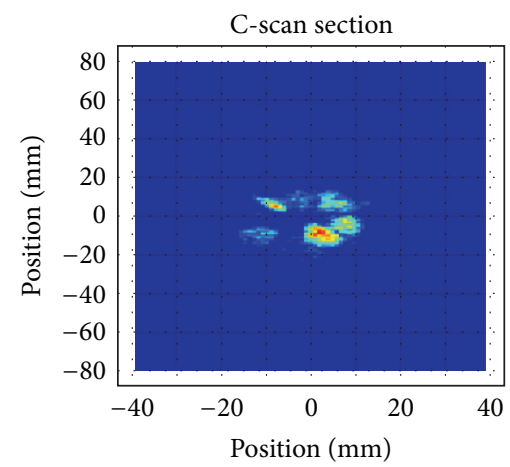

(b) $43-69 \mathrm{ps}$

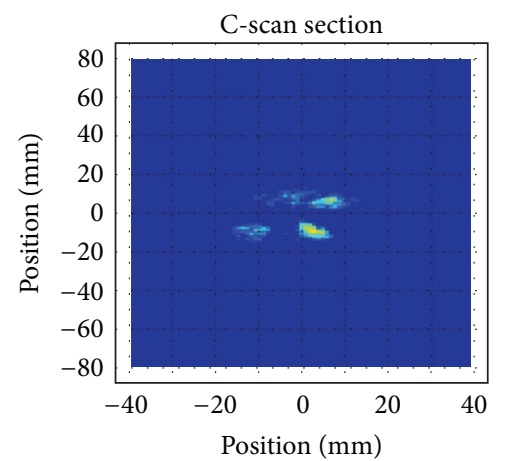

(c) $47-69 \mathrm{ps}$

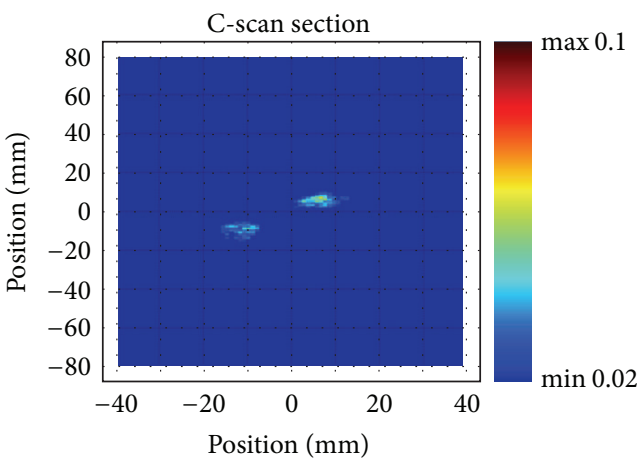

(d) $56-69 \mathrm{ps}$

FIGURE 8: Time-domain TDS waveform of glass composite laminate (a) and impact delamination images using different time gates (from left: (b) 43-69 ps, (c) 47-69 ps, and (d) 56-69 ps). 


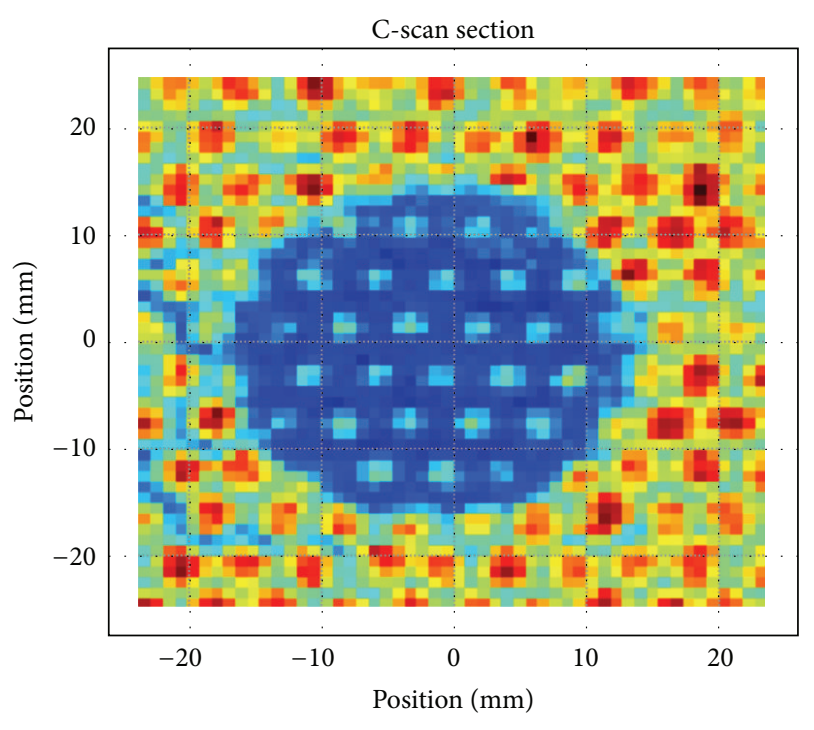

(a) C-scan image

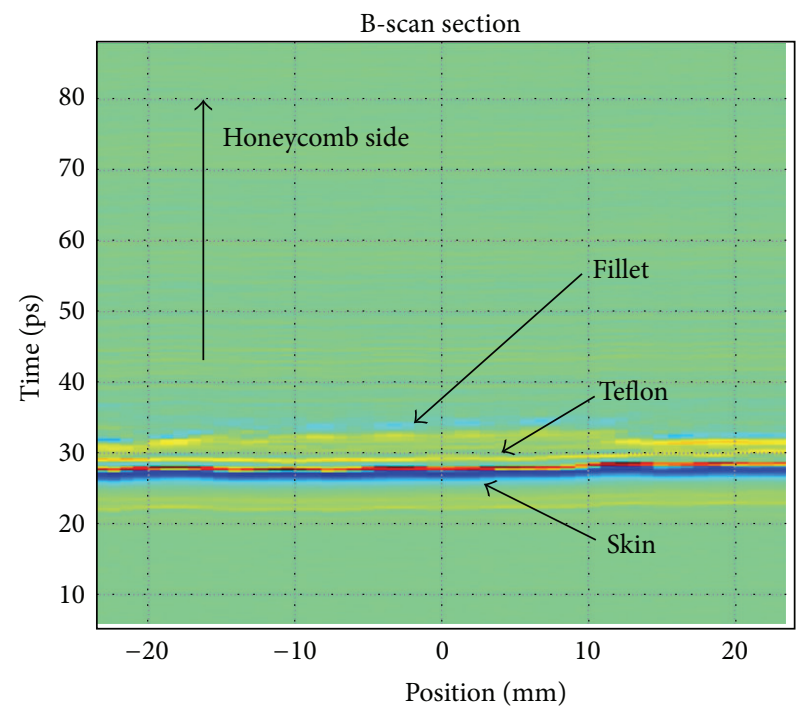

(b) B-scan image

Figure 9: Embedded defects in Kevlar/Nomex honeycomb sandwich. (a) C-scan image based on amplitude and (b) B-scan image in the horizontal middle location of image (a).

using the frequency-domain FFT signal. Figure 9 shows the T-ray images of the reflected signal from the top facesheet of the Kevlar/Nomex panel, together with the image based on the peak amplitude of the peak-to-peak amplitude signal. The circle on the image was a $25.4 \mathrm{~mm}$ diameter double Teflon film beneath the facesheet, and the B-scan image on (a) was made. Also, fillet, Teflon film, and skin were easily observed as an ultrasonic method.

3.3. E-Field Characterization in Conducting Carbon Fiber. Terahertz waves can penetrate dielectric materials quite easily but not electrically conducting materials. Also, the application of electrical resistance technique could be found for impact damage detection of multilayer woven carbon composite [8], cross-ply CFRP [9], unidirectional CFRP [10], and randomly distributed short/nanotube carbon fibers [1012]. The application of terahertz waves to the inspection of carbon composites is still under progress in the literature [10]. Carbon fiber-reinforced polymer (CFRP) composites are poor conductors for electricity, and the conductivity is anisotropic (unidirectional, 33\% resin content and $3.08 \mathrm{~mm}$ in thickness); so, it is worthwhile to quantify the penetration of terahertz waves in carbon composites. The carbon fibers used in the manufacturing of CFRP are highly anisotropic microscopically; the electrical conductivity along the fiber axis is about three orders of magnitude greater than that in the radial direction. In a unidirectional laminate of carbon fiber composite, the transverse electrical conductivity is further impeded by the lack of continuity.

The conduction mechanism in the transverse direction (perpendicular to the fiber axis) is a percolation process that relies on the random contact between adjacent fibers. In the literature, the electrical conductivity data for carbon composites are somewhat sparse [13]. The reported values of longitudinal conductivity $\left(\sigma_{l}\right)$ varied from $1 \times 10^{4} \mathrm{~S} / \mathrm{m}$ to $6 \times 10^{4} \mathrm{~S} / \mathrm{m}$. The range of reported data on the transverse conductivity $\left(\sigma_{t}\right)$ is particularly large, from $2 \mathrm{~S} / \mathrm{m}$ to as high as $600 \mathrm{~S} / \mathrm{m}$.

The value of transverse conductivity in a unidirectional laminate is highly dependent on the manufacturing process and the quality of the composite. In a unidirectional carbon composite, the in-plane conductivity with the electrical current flowing at an angle $\theta$ from the fiber axis is given by [14] as follows:

$$
\sigma=\sigma_{l} \cos ^{2} \theta+\sigma_{t} \sin ^{2} \theta
$$

Because of the highly anisotropic electrical conductivity $\left(\sigma_{l} \gg \sigma_{t}\right)$, the penetration of terahertz waves through a unidirectional carbon composite depends on the relative angle between the electrical field vector and the fiber axis. Using a value of $\sigma_{t}=10 \mathrm{~S} / \mathrm{m}$, the skin depth of a unidirectional carbon composite for a terahertz wave, with the electric field oriented normal to the fiber axis, is approximately $0.2 \mathrm{~mm}$ at $1 \mathrm{THz}$ and $0.5 \mathrm{~mm}$ at $0.1 \mathrm{THz}$. Experimentally, we have measured the angular dependence of the power transmission through a 22-ply unidirectional carbon composite laminate using the CW terahertz system. Near the low end of the frequency spectrum $(f \sim 0.1 \mathrm{THz})$, the transmitted power is more than $30 \mathrm{~dB}$ above the noise floor. The angular dependence of the transmitted power at $0.1 \mathrm{THz}$ is shown in Figure 10.

When compared to the theory prediction based on the angular-dependent conductivity, the measured power transmission at angles away from 90 degrees much higher this predicted. The value would have the unidirectional carbon composites behaving like a polarizer with a sharp cut-off under the assumptions that the incident terahertz ray is linearly polarized and that the fiber axes in the laminate are all parallel. It seems that the discrepancy contributes to the previous involved things. 


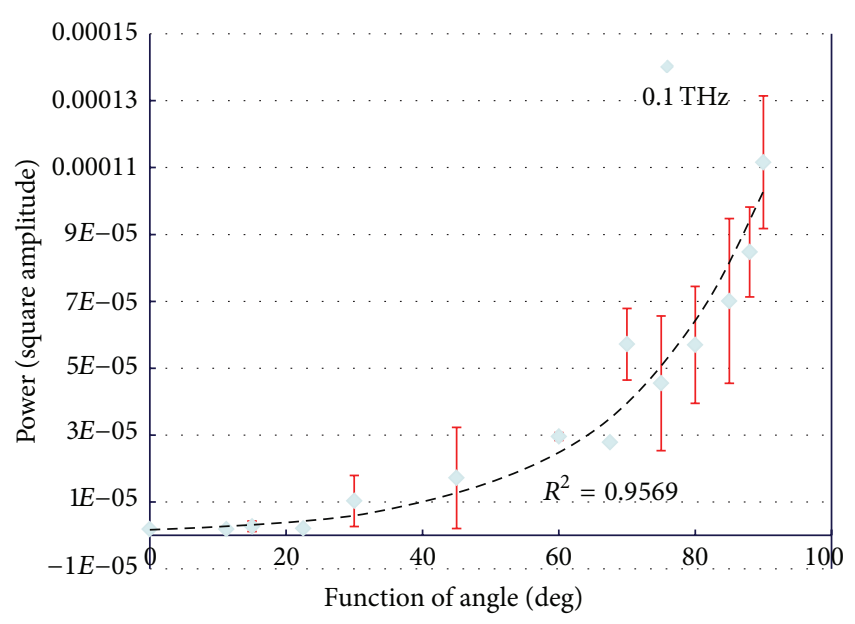

FIgURE 10: Angular dependence of transmitted power of $\mathrm{THz}$ terahertz waves through a 22-ply unidirectional carbon composite laminate.

TABLE 2: Modeled conductivity of the first ply.

\begin{tabular}{lccccc}
\hline & $\mathrm{S} \operatorname{can} 0^{\circ}$ & $\mathrm{S} \operatorname{can} 22.5^{\circ}$ & $\mathrm{S} \operatorname{can} 45.0^{\circ}$ & $\mathrm{S} \operatorname{can} 67.5^{\circ}$ & $\mathrm{S} \operatorname{can} 90^{\circ}$ \\
\hline$\theta$ & 0 & 22.5 & 45 & 67.5 & 90 \\
$\sigma_{1}$ & 1.0 & 0.85 & 0.5 & 0.15 & 0 \\
$\sigma$ & $1.0 \cos ^{2} \theta$ & $0.85 \cos ^{2} \theta$ & $0.5 \cos ^{2} \theta$ & $0.15 \cos ^{2} \theta$ & $0 \cos ^{2} \theta$ \\
$R_{\mathrm{eq}}$ & 1 & 0.73 & 0.25 & 0.022 & 0 \\
\hline
\end{tabular}

3.4. THZ Images in Conducting CFRP Solid Laminate. A reflective TDS system was utilized in order to evaluate the effect of $E$-filed direction on PPS CFRP solid laminate. A PPS CFRP solid laminate was scanned at different angles.

Sample's layup is $[45 / 0 /-45 / 90]_{6 \mathrm{~s}}$ and $114 \times 355 \times 6.8 \mathrm{~mm}$ in size (resin content: $33 \%$ ). Figure 11 shows a sample setup of TDS system for scanning. Figure 12 shows a scan configuration and direction of $E$-field normal to fiber, and $\theta$ is the angle between fiber direction and $E$-field direction. Figure 12 shows TDS scan images at the angles $(\theta)$ of $-22.5^{\circ}, 90.0^{\circ}, 22.5^{\circ}, 45.0^{\circ}$, $67.5^{\circ}$, and $0^{\circ}$. These images were mapped out based on the $E$ field direction in the CFRP solid laminates. Scan size is $60 \times$ $60 \mathrm{~mm}$, and step size is $0.5 \mathrm{~mm}$. These images are based on a reflection mode at the scan range of $\Delta t$ (scan gate length) $=10$ ps. Especially, at $\theta=-45^{\circ}$, this image was observed to a clear and higher contrast image if compared with the rest cases, $\theta=-22.5^{\circ}, 22.5,45.0,67.5$, and $0^{\circ}$. Here, there are some lines in the angle of $\theta=-45^{\circ}$ due to the $E$-filed direction of $\mathrm{THz}$ wave. It is thought that the $\mathrm{THz}$ wave penetrated a couple of a CFRP prepreg sheet called a scan depth. Table 2 shows the simple modeled data on the $E$-felid direction to the sample.

As described easlier, being based on the electrical conductivity in carbon composites, we tried to understand the penetration of fiber orientation in a carbon composite laminate. As shown in Figure 12, the electric field (E-field) direction is horizontal, the fiber direction is vertical, and the difference angle is called $\theta$. Here, by using TDS terahertz

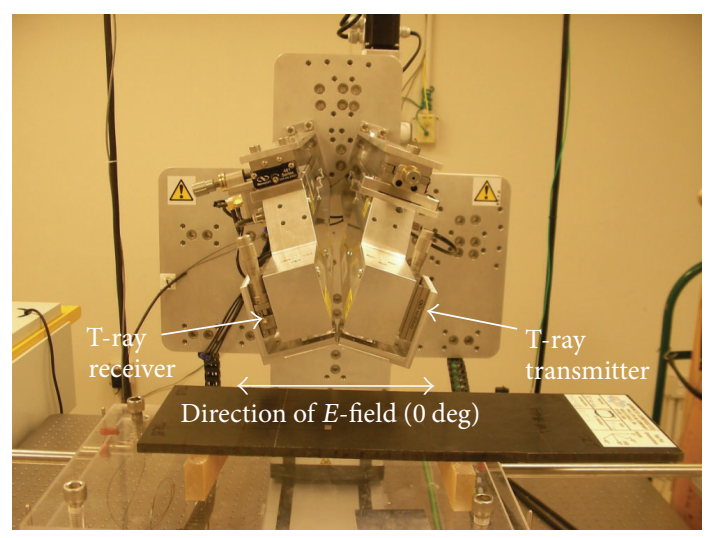

FIGURE 11: Diagram showing a sample set up system.

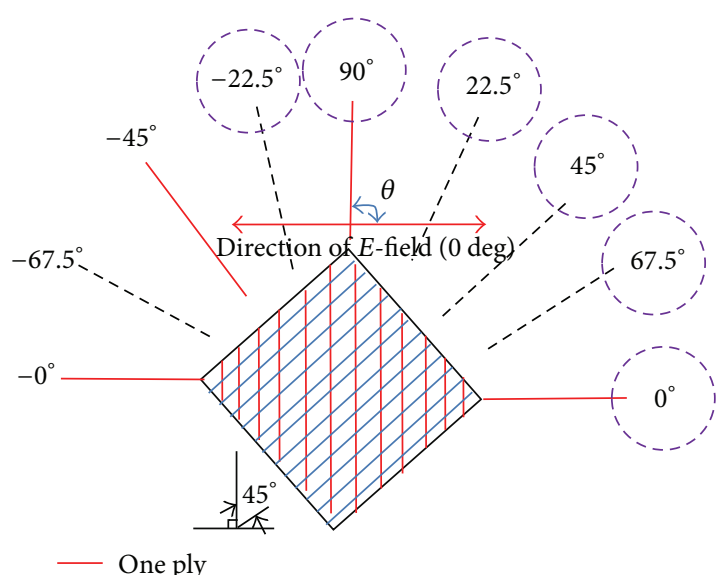

FIGURE 12: Diagram showing a scan configuration of TDS for scanning and direction of $E$-field normal to fiber, and $\theta$ is the angle between fiber direction and $E$-field direction.

waves in the reflection mode, the flaws were scanned and imaged.

We then correlated the $\mathrm{S} / \mathrm{N}$ of the flaw images to the conductivity of the first two plies. Since $\sigma_{l} \gg \sigma_{t}$, (7) may be written as $\sigma \approx \sigma_{l} \cos ^{2} \theta$. To interpret the T-ray scan images of the flaws under one ply, one must consider electric field ( $E$-field) direction of the T-ray and the lay-up direction of CFRP composite laminates. First, the penetration through the one ply depends on the relative angle between the $E$ field and the fiber direction in the one ply. Secondly, one should consider the fact that the radiation is focused on the surface of the sample. A simple argument is just needed as one resistor so that the resistance $R$ is given by $(1 / R)=$ $\left(1 / R_{1}\right)[14]$. For this equation, we get the conductivity $\sigma_{1}$ and correlate its conductivity noise ratio of the flaws in the Tray scan image. By using $\sigma \approx \sigma_{t} \cos ^{2} \theta$, the conductivity has the lowest value $\left(\approx 0.0 \sigma_{t}\right)$ when the $E$-field makes 90 degrees. On one ply to the $E$-field direction. On one ply to the E-field direction, the $\mathrm{S} / \mathrm{N}$ of the flaw image is therefore expected to be the highest when the sample is at this angle. In contrast, sample direction that gave a high value conductivity, such as $\theta=0$ degrees $\left(\sigma=1.0 \sigma_{t}\right)$, had poor signal-to-noise ratio. 

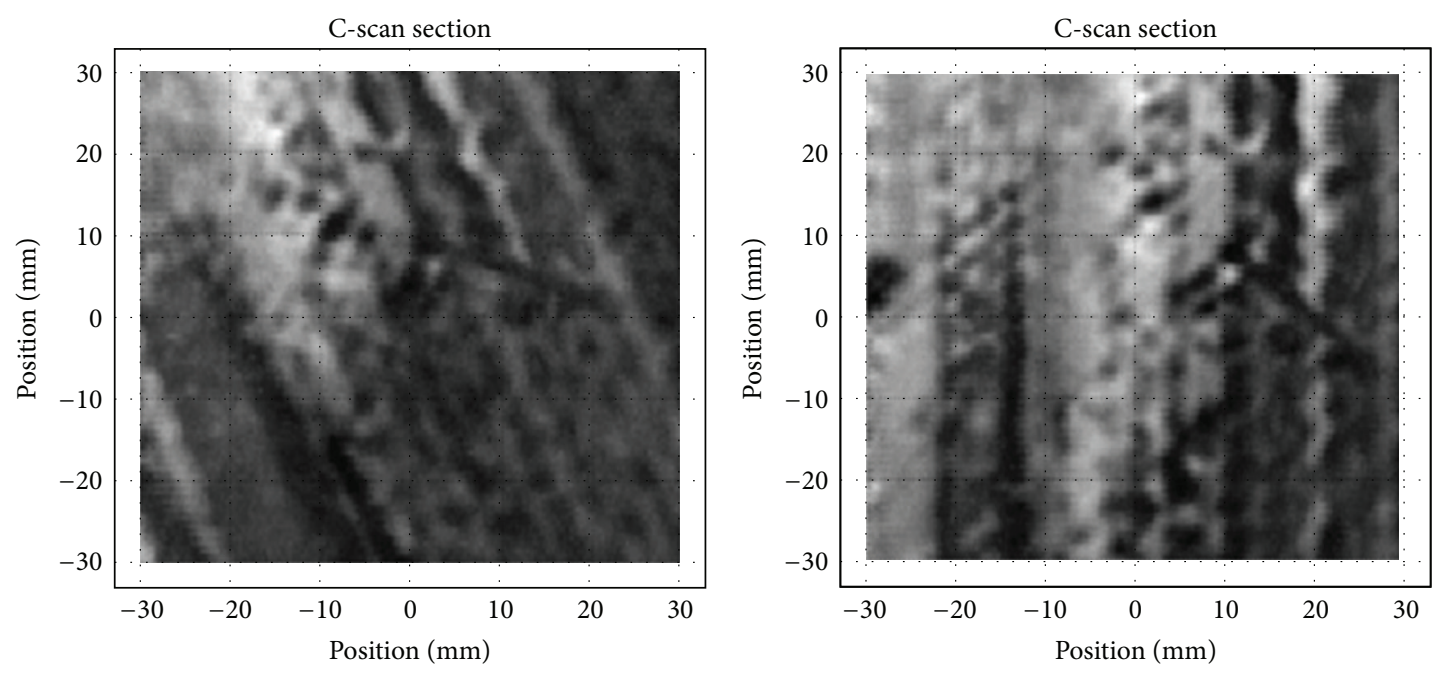

(a) $\theta=-22.5^{\circ}$

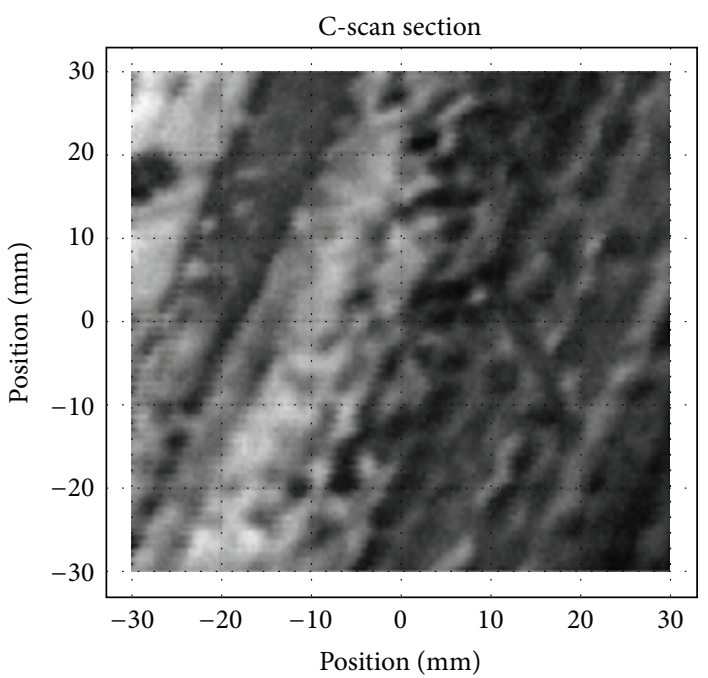

(b) $\theta=90^{\circ}$

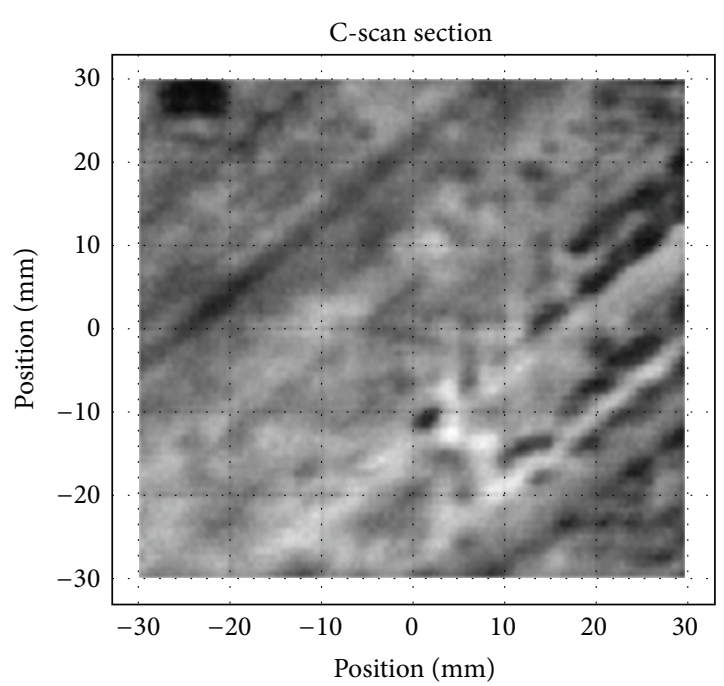

(c) $\theta=67.5^{\circ}$

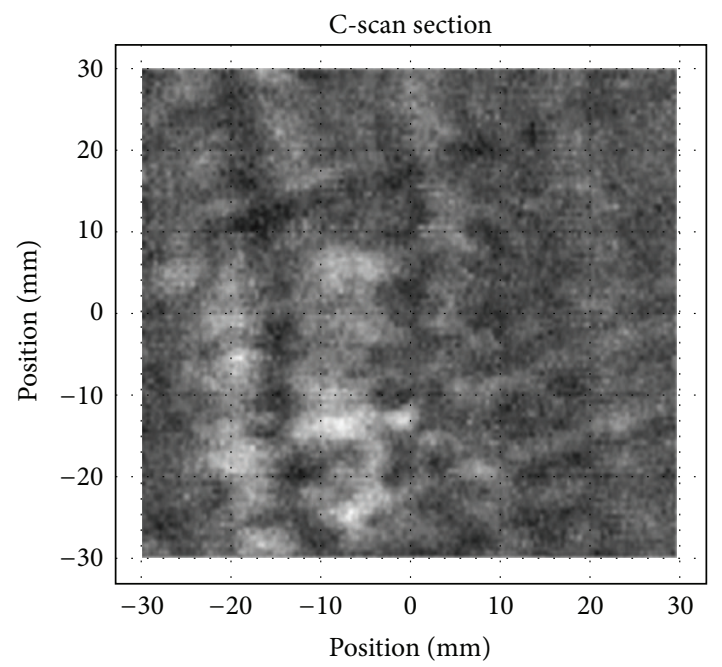

(d) $\theta=45.0^{\circ}$

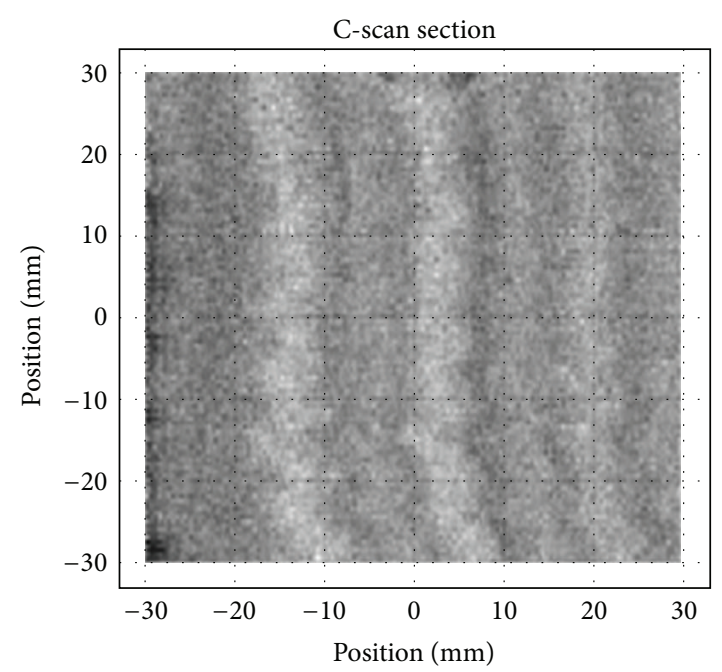

(e) $\theta=22.5^{\circ}$

(f) $\theta=0.0^{\circ}$

FIgURE 13: Diagram showing TDS scan images at the angles $(\theta)$ of $-0^{\circ},-45^{\circ}, 90^{\circ}$, and $0^{\circ}$. 


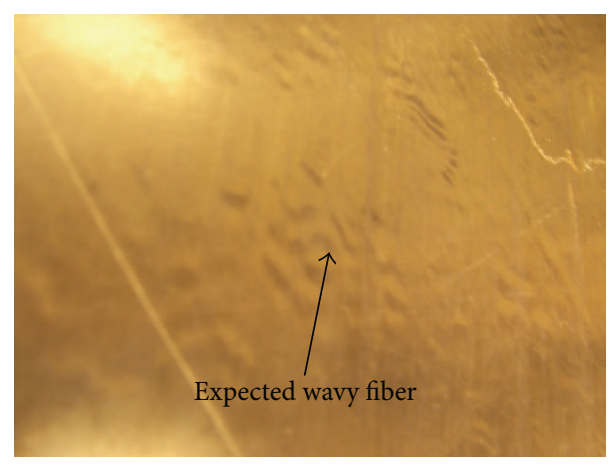

(a) Photo on the surface of the sample

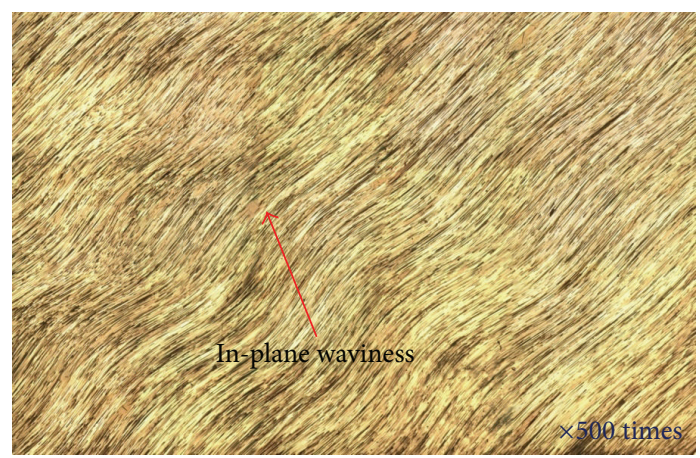

(b) Macroscopic photo on waviness

Figure 14: Photo of sample surface and microscopic photo of inplane fiber waviness in CFRP composites.

A trend of fiber penetration shows that the prediction of $\mathrm{S} / \mathrm{N}$ based on the conductivity is in qualitative agreement with the testing. So, it is found that the penetration of the fiber depends on the relation between the $E$-field and the fiber direction. So, Figure 13 shows the reflection mode images under the different angle between the $E$-field and the fiber direction. So, especially, Figure 13(b) shows the highest S/N T-ray image; however, Figure 13(f) shows the lowest S/N T-ray image. Therefore, Figure 13(b) shows the fiber orientation $\left(90^{\circ}\right.$ and $45^{\circ}$ ) of two plies in CFRP laminates due to best optimization in a function of fiber angle; however, Figure 13(f) shows no information in fiber orientation due to the poor angle optimization because the fiber is blocking the T-ray. The dots of T-ray images were observed in Figures 13(a), 13(b), 13(c), and 13(d). A photo was taken in some part in sample as shown in Figure 14(a) which shows dark spots in some areas. In-plane waviness was observed in Figure 14(b); so, such waviness seems to be related to the fiber angles. It was found that the dots in T-ray images were caused by the different angles in the fibers. It is needed to consider the fiber direction in order to quantify the penetration of terahertz waves in carbon composites.

\section{Conclusions}

Initial results were obtained in an exploration of terahertz wave applications for the NDT of composites. A composite with various thicknesses was examined using a THz TDS system. Index of refraction in the terahertz frequency range for composite was measured using a reflection and transmission configuration. THz TDS reflection imaging on the composite with flaw was performed.

First of all, it was also easier to measure the refractive index of the composite using a transmission configuration. A reflection mode is certainly a more likely case for a maintenance technique. And, in non-conducting composites of glass, Kevlar/Nomex sandwich panels, Fused quartz, and PMMA, terahertz can complement ultrasonic NDT, especially with its penetration ability. The conductivity of carbon fibers will substantially limit the utility of terahertz waves even in one- or two-ply PPS CFRP and CFRP laminates; therefore, it is found that the penetration of the fiber depends on the relation between the $E$-field and the fiber direction. It was found that the various dots in T-ray images were caused by the different angles in the fibers. It is thought that an application of terahertz applications for composite NDT could be very useful. Also, terahertz radiation can exhibit some unique characteristics for nondestructive evaluation form other forms of electromagnetic radiation.

\section{Acknowledgment}

This research was supported by Basic Science Research Program through the National Research Foundation of Korea (NRF) funded by the Ministry of Education, Science and Technology (no. 2011-0008391).

\section{References}

[1] C. P. Chiou, J. L. Blackshire, R. B. Thompson, and B. B. Hu, "Terahertz ray system calibration and material characterzations," Review of QNDE, vol. 28, pp. 410-417, 2009.

[2] R. Huber, A. Brodschelm, F. Tauser, and A. Leitenstorfer, "Generation and field-resolved detection of femtosecond electromagnetic pulses tunable up to $41 \mathrm{THz}$, , Applied Physics Letters, vol. 76, no. 22, pp. 3191-3193, 2000.

[3] J. V. Rudd and D. M. Mittleman, "Influence of substrate-lens design in terahertz time-domain spectroscopy," Journal of the Optical Society of America B, vol. 19, no. 2, pp. 319-329, 2000.

[4] I. S. Gregory, C. Baker, W. R. Tribe et al., "Optimization of photomixers and antennas for continuous-wave terahertz emission," IEEE Journal of Quantum Electronics, vol. 41, no. 5, pp. 717-728, 2005.

[5] E. R. Brown, F. W. Smith, and K. A. McIntosh, "Coherent millimeter-wave generation by heterodyne conversion in lowtemperature-grown GaAs photoconductors," Journal of Applied Physics, vol. 73, no. 3, pp. 1480-1484, 1993.

[6] E. R. Brown, K. A. McIntosh, K. B. Nichols, and C. L. Dennis, "Photomixing up to $3.8 \mathrm{THz}$ in low-temperature-grown GaAs," Applied Physics Letters, vol. 66, no. 3, pp. 285-287, 1995.

[7] D. . Mittleman, R. H. Jacobsen, and M. C. Nuss, "Tray imaging," IEEE Journal of Selected Topics in Quantum Electronics, vol. 2, pp. 679-689, 1996.

[8] N. Angelidis, N. Khemiri, and P. E. Irving, "Experimental and finite element study of the electrical potential technique for damage detection in CFRP laminates," Smart Materials and Structures, vol. 14, no. 1, pp. 147-154, 2005. 
[9] L. Hou and S. A. Hayes, "A resistance-based damage location sensor for carbonfiber composites," Smart Materials and Structures, vol. 11, pp. 966-969, 2002.

[10] R. Schueler, S. P. Joshi, and K. Schulte, "Damage detection in CFRP by electrical conductivity mapping," Composites Science and Technology, vol. 61, no. 6, pp. 921-930, 2001.

[11] C. Bois and C. Hochard, "Measurements and modeling for the monitoring of damaged laminate composite structures," in Proceedings of the 1st European Workshop on Structural Health Monitoring (EWSHM '02), pp. 425-432, 2002.

[12] S. Kirkpatrick, "Percolation and conduction," Reviews of Modern Physics, vol. 45, pp. 574-588, 1973.

[13] D. K. . Hsu, "Characterization of a graphite/epoxy laminate by electrical resistivity measurements," Review of Progress in Quantitative NDE, vol. 4, pp. 1219-1228, 1985.

[14] K. W. Tse, C. A. Moyer, and S. Arajs, "Electrical conductivity of graphite fiber-epoxy resin composites," Materials Science and Engineering, vol. 49, no. 1, pp. 41-46, 1981. 

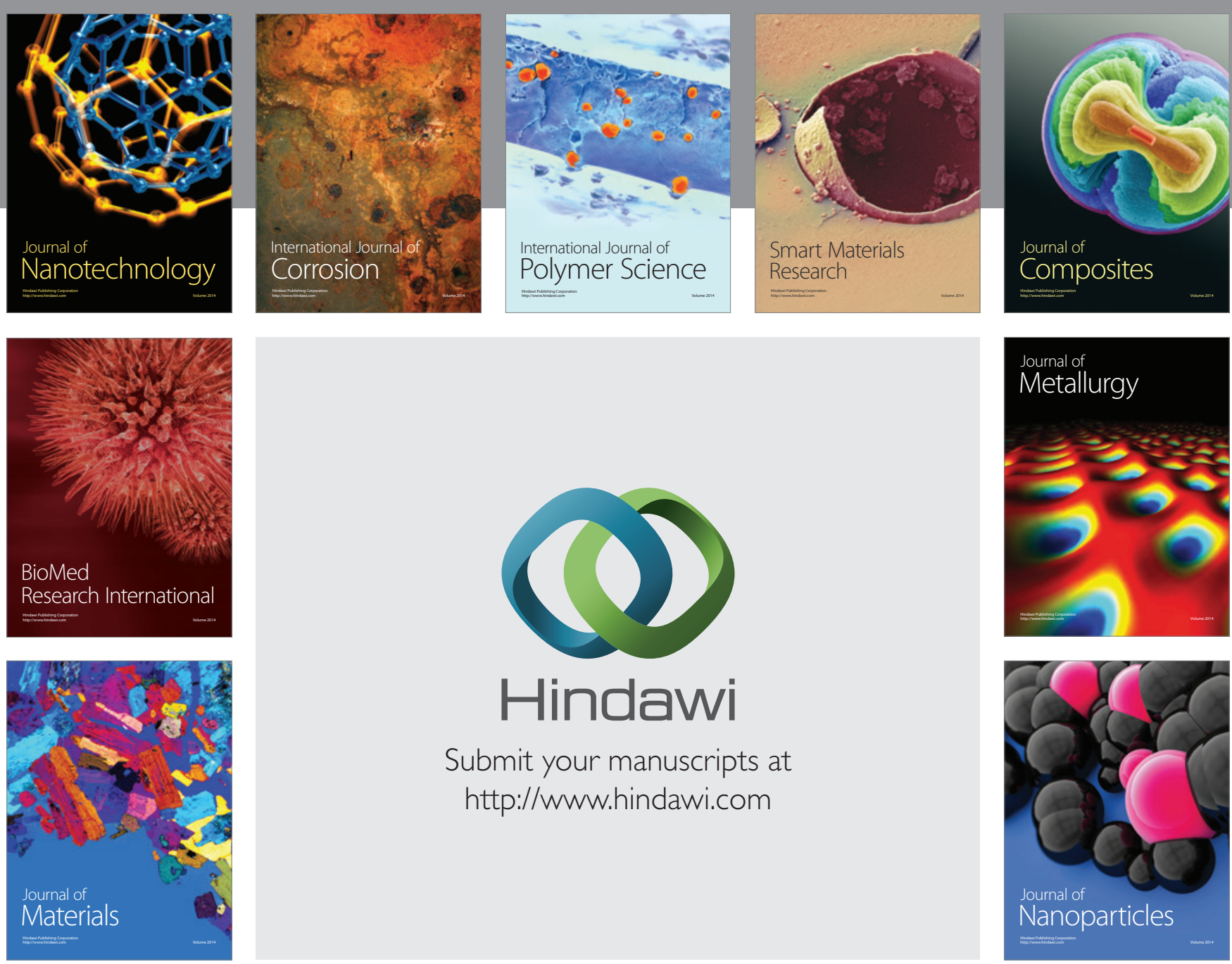

Submit your manuscripts at http://www.hindawi.com
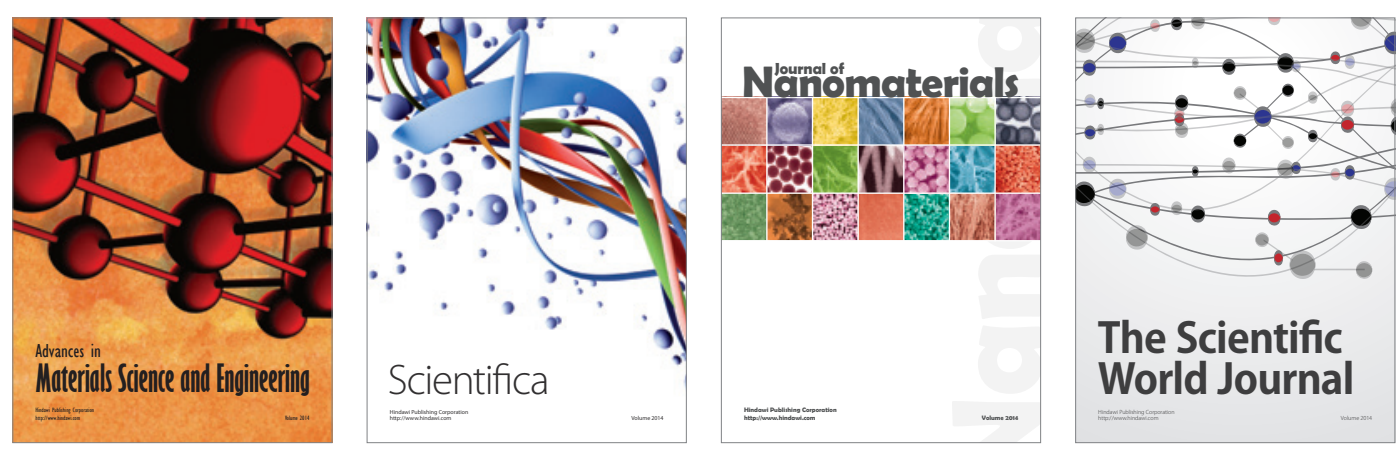

\section{The Scientific World Journal}
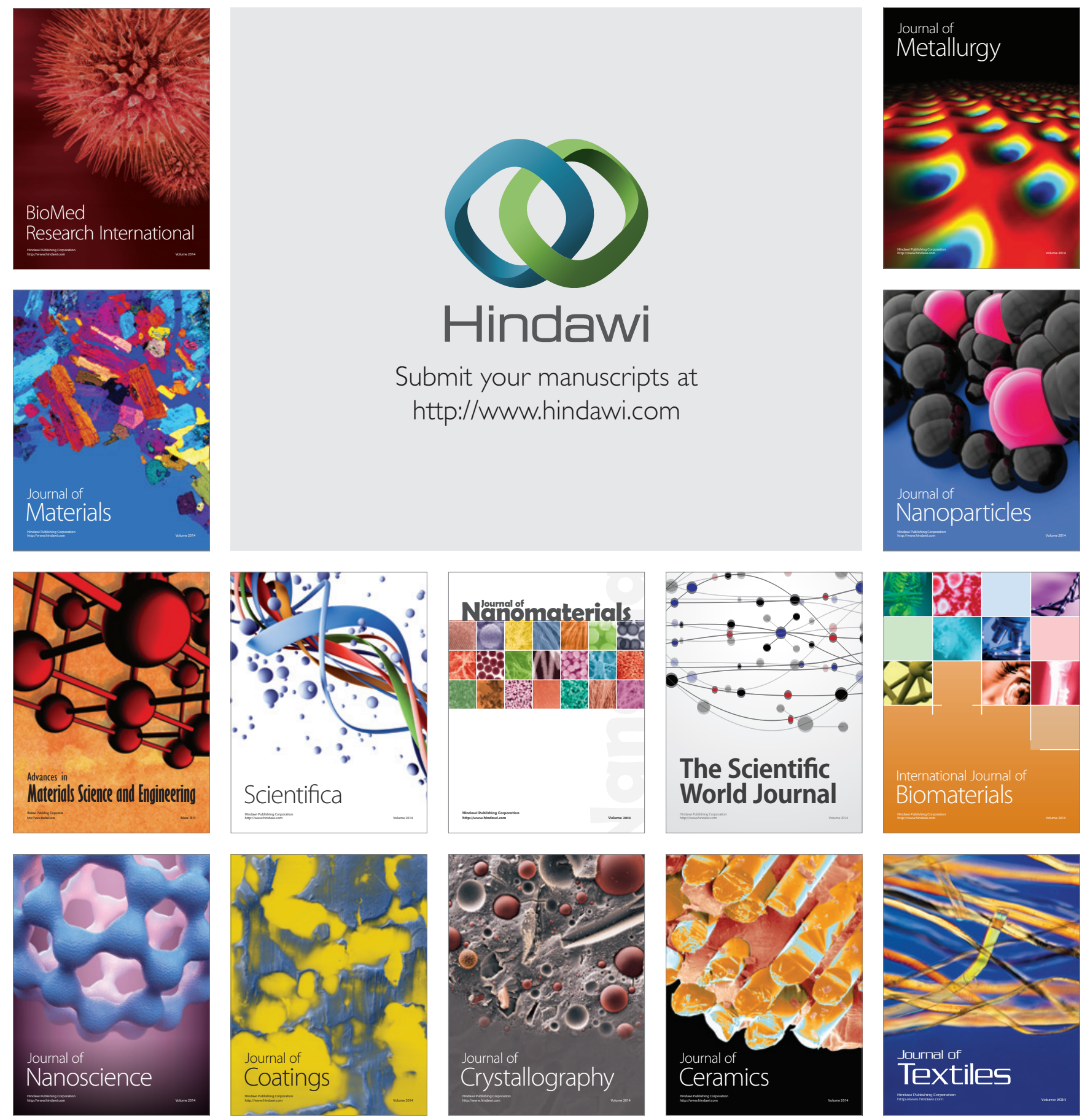